\title{
Exceptional points in Fermi liquids with quadrupolar interactions
}

\author{
Rui Aquino ${ }^{1}$ and Daniel G. Barci ${ }^{1}$ \\ ${ }^{1}$ Departamento de Física Teórica, Universidade do Estado do Rio de Janeiro, \\ Rua São Francisco Xavier 524, 20550-013 Rio de Janeiro, RJ, Brazil
}

(Dated: November 3, 2020)

\begin{abstract}
We show the existence of non-Hermitian degeneracies, known as exceptional points, in the collective mode spectrum of Fermi liquids with quadrupolar interactions. Through a careful analysis of the analytic properties of the dynamic quadrupolar susceptibility, we show that, in the weak attractive region, two stable collective modes coalesce to an exceptional point. We completely characterize this singularity, explicitly showing its topological properties. Experimental signatures are also discussed.
\end{abstract}

Introduction- Open quantum systems play a central role in most applications of quantum mechanics ${ }^{1}$. An important theoretical tool to describe dissipative quantum systems is the modelling of locally non-conservative systems by effective non-Hermitian Hamiltonians $\mathrm{2}^{-4}$. These types of Hamiltonians has several counterintuitive properties. Perhaps, one of the most streaking ones is the appearance of non-Hermitian degeneracies ${ }^{5}$ known as exceptional points (EP) ${ }^{6.7}$.

When a non-Hermitian Hamiltonian continuously depends on external parameters, it could happen that, for certain values of the parameters, two or more eigenvalues coalesce to an EP. However, this is not a usual degeneracy, as observed in Hermitian systems. In an EP, not only the eigenvalues coincide but also the eigenvectors become linearly dependent ${ }^{8}$, reducing in this way the dimension of the subspace associated to the degenerated eigenvalue. This singularity of the Hilbert space has remarkable topological consequences ${ }^{9-13}$. The relation between EPs and dynamical phase transitions was early recognized in theoretical as well as experimental works 14 -17 .

In recent years, exciting findings of EPs are shown up in very different contexts, strengthening the broad interest of this subject; from nuclear $\frac{18}{}$ and atomic physics $\frac{19-21}{}$ to Bose-Einstein condensates 22 and strongly correlated fermion systems ${ }^{23}$, passing through microwave cavities ${ }^{24}$ and SWAP gates in spin systems $\frac{16,25}{}$. Moreover, topological properties of EPs were experimentally studied in metamaterials set ups ${ }^{26,27}$.

In this letter, we report the existence of exceptional points in the spectrum of collective excitations of Fermi liquids ${ }^{28}$ with higher order Landau parameter interactions. Fermi liquids with quadrupolar interactions began to call the attention of the condensed matter community because it is the simplest model supporting an isotropic-nematic transition $\stackrel{1}{1}$. Nematic fluctuations play a crucial role in several strongly correlated systems, such as cuprates and Fe-based superconductors and a variety of Quantum Hall Effects $\frac{30}{}$.

Collective excitations of Fermi liquids with quadrupolar interactions have been studied in different regimes 1,2,32-35. Here, we explicitly show the appearance of a non-Hermitian singularity for weak quadrupolar attraction. We completely characterize this exceptional point, by analyzing the Hilbert space structure and its topological properties. Finally, we discuss some possible experimental set ups.

Model- We consider the simplest model of bi-dimensional spinless Fermions with local quadrupolar interactions. The
Hamiltonian is

$$
H=\int d^{2} r\left\{\psi^{\dagger}(\mathbf{r}) \epsilon(\nabla) \psi(\mathbf{r})+\frac{F_{2}}{4} \operatorname{Tr}\left[Q^{2}(\mathbf{r})\right]\right\}
$$

where $\psi(r)$ is a spinless Fermionic field operator. The bare dispersion relation is given by $\epsilon(\nabla)$, where $\nabla$ is the twodimensional gradient operator. $F_{2}$ is the quadrupolar coupling constant. The quadrupolar Fermionic density $Q_{i j}=$ $\psi^{\dagger}(\mathbf{r})\left[\nabla_{i} \nabla_{j}-\left(\delta_{i j} / 2\right) \nabla^{2}\right] \psi(\mathbf{r})$, with $i=1,2$, is a symmetric traceless tensor of rank 2 , invariant under $\pi$ rotations.

Collective modes are encoded in the dynamic quadrupolar susceptibility (DQS) $\chi_{i j l m}(\omega, \mathbf{q})=$ $\left\langle Q_{i j}(-\omega,-\mathbf{q}) Q_{l m}(\omega, \mathbf{q})\right\rangle$. DQS have been intensively studied $1,2,36,37$ in the vicinity of a quantum critical point, where non-Fermi liquid behavior is espected. Conversely, in this letter we study the dynamic response in the Fermi liquid regime. Since the quadrupolar moment has two degrees of freedom, the susceptibility has essentially two independent polarizations, the longitudinal $\chi_{2}^{+}(\omega, \mathbf{q})$ and the transversal polarization $\chi_{2}^{-}(\omega, \mathbf{q})$. These quantities have been computed using different approximation approaches $1,2,32,33,38$. In the limit of small momentum $q<<k_{F}$, where $k_{F}$ is the Fermi momentum, the result is ${ }^{2}$ (please, see supplemental material for a detailed description of the calculation)

$$
\chi_{2}^{ \pm}(\omega, \mathbf{q})=\frac{\chi_{0}^{0}(s) \pm \chi_{4}^{0}(s)}{1-F_{2}\left(\chi_{0}^{0}(s) \pm \chi_{4}^{0}(s)\right)}
$$

where

$$
\chi_{2 \ell}^{0}=\left[-\delta_{\ell, 0}+K_{0}(s)\left(\frac{1-K_{0}(s)}{1+K_{0}(s)}\right)^{\ell}\right]
$$

with $K_{0}(s)=s / \sqrt{s^{2}-1}$. Eq. (3) with $\ell=0,2$ are the bare density and quadrupolar susceptibilities, respectively. Eq (S26) has the usual structure of an effective interaction in the traditional RPA approximation. Due to the locality of the quadrupolar interaction (i.e.,$F_{2}$ do not depend on q), the DQS is not a function of $\omega$ and $\mathbf{q}$ independently. Instead, it depends on the dimensionless variable $s=\omega / q v_{F}$, where $\omega$ is the frequency and $q v_{F}$ is the maximum energy of a particlehole excitation with momentum $q=|\mathbf{q}|$ and Fermi velocity $v_{F}=\left|\mathbf{v}_{\mathbf{F}}\right|$. It is worth mentioning that in the computation of Eq. (S26), rotational invariance and particle-hole symmetry were imposed. 
Collective modes- The DQS is an analytic function of $s$, having poles and cuts. It has branch points at $s=$ \pm 1 ; the threshold of Landau damping $\omega= \pm v_{F} q$. We will focus on the longitudinal polarization $\chi_{2}^{+}(s)$ since, as we will show, this component displays an EP. Collective modes are computed by solving the algebraic equation $F_{2}\left(\chi_{0}^{0}(s)+\chi_{4}^{0}(s)\right)=1$. We have numerically solved it for $F_{2}$ running from the strong attractive $\left(F_{2}=-1\right)$ to the strong repulsive regime $\left(F_{2}>1\right)$. We display the result in Fig. (1). In
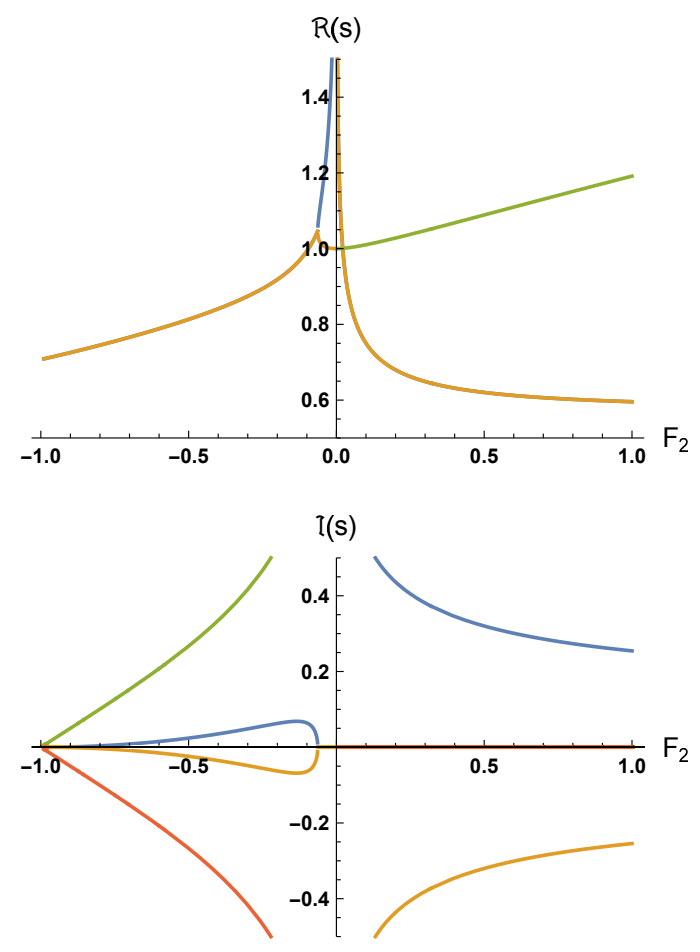

FIG. 1. Collective modes from the longitudinal polarized component of the DQS $\chi_{2}^{+}(s)$. In the upper panel we plot $\Re\left[s\left(F_{2}\right)\right]$ while in the lower panel we depict $\Im\left[s\left(F_{2}\right)\right]$.

the upper panel, we show the real part of the collective modes as function of $F_{2}$, while in lower panel, we show the imaginary part. In the repulsive region $\left(F_{2}>0\right)$, we observe a stable (real) mode that tends to $s=1$ when $F_{2} \rightarrow 0$. This is the quadrupolar equivalent of the Landau zero sound. In addition, a damped mode also appears in the same region. The stable mode is continuously extended to the weak attractive region $F_{2} \lesssim 0$. However, in this regime, there is another stable mode with a divergent behavior, $s \rightarrow+\infty$ when $F_{2} \rightarrow 0^{-}$. The existence of such a mode was reported in Ref. 33. Interestingly, there is a special point, $F_{2}^{c}$, where both stable modes meet together. For $F_{2}<F_{2}^{c}$, these modes become damped as can be clearly seen in the lower panel of Fig. 1. We can also observe an overdamped mode (purely imaginary) in all the attractive region. This mode is the precursor of the isotropic-nematic phase transition that occurs at $F_{2}=-1$ and has already been extensively studied ${ }^{1.2}$.

Exceptional point- In order to analytically characterize the singularity at $F_{2}=F_{2}^{c}$, we first observe that $s\left(F_{2}^{c}\right) \gtrsim 1$, being well separated from the cut $s^{2}<1$. On the other hand, the singularity is sufficiently close to $s=1$, allowing us to try a series expansion of $\chi_{2}^{+}(s)$ in the neighborhood of $s=1$. For simplicity, let us work with the inverse of the DQS, $\mathcal{L}^{+}(s)=\left(\chi_{2}^{+}(s)\right)^{-1}$. Expanding this quantity in terms of the variable $\sqrt{(s-1) / 2}$, we find the following expansion (please, see supplemental material for details of the calculation),

$$
\mathcal{L}^{+}(s)=-F_{2}+\sqrt{\frac{s-1}{2}}+5\left(\frac{s-1}{2}\right)+O\left((s-1)^{3 / 2}\right) .
$$

Longitudinal quadrupolar fluctuations $\delta Q^{+}(s, \mathbf{q})$ are governed by the effective action

$$
S_{\text {eff }}=\int \frac{d \omega d^{2} q}{(2 \pi)^{3}} \mathcal{L}^{+}(s)\left|\delta Q^{+}(s, \mathbf{q})\right|^{2} .
$$

The collective modes are given by the roots of $\mathcal{L}^{+}(s)=0$. Using Eq. (S30), we obtain

$$
s_{ \pm}=\frac{1}{25}\left\{\left(26+10 F_{2}\right) \pm \sqrt{20 F_{2}+1}\right\} .
$$

$s_{ \pm}\left(F_{2}\right)$ have a square root singularity (branch point) at $F_{2}^{c}=$ $-1 / 20$. At this point, both zeros are degenerated, $s_{ \pm}\left(F_{2}^{c}\right)=$ $51 / 50$. We depict the real and imaginary part of $s_{ \pm}\left(F_{2}\right)$ in Fig. (2). Thus, the approximation made in Eq. (S30) for $\mid s-$
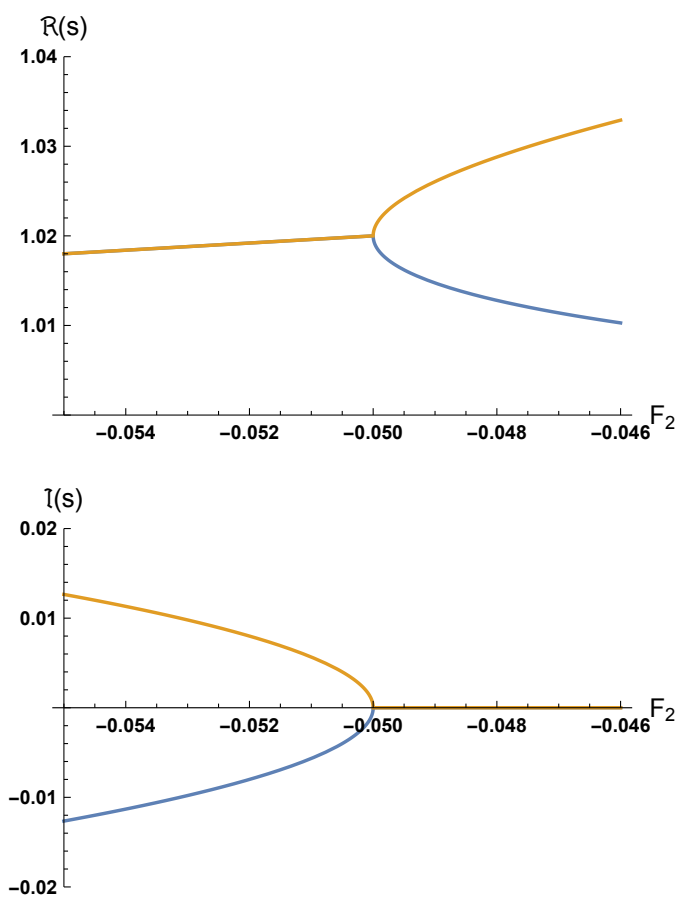

FIG. 2. Solutions of $\mathcal{L}^{+}\left(s_{ \pm}\right)=0$, given by Eq. S31 as a function of the parameter $F_{2}$. The upper panel shows the real part of $s_{ \pm}$, while lower one depicts the imaginary part. The point $F_{2}=-1 / 20$, where both eigenvalues coalesce and the imaginary part emerges, is the exceptional point.

$1 \mid<<1$, correctly captures the presence of the degeneracy 
point observed in the numerical computation of Fig. (1). The square-root singularity is a typical signature of an exceptional point $\frac{39}{}$.

The dynamics described by Eq. (S30) is non-local in time. However, since the degeneracy is separated from the cut, we can further expand $\mathcal{L}^{+}(s)$ in the neighborhood of $s=s_{ \pm}$. In addition, we observe that the local character of the interaction imposes that $\mathcal{L}^{+}(s)$ only depends on the dimensionless variable $s$. Thus, we can consider quadrupolar fluctuations, $\delta Q^{+}(s)$, ignoring any momentum dependence not scaling with $s$. The consequence is that all collective modes in this approximation have linear dispersion relation $\omega \sim v_{F} q$. This is a good approximation for weak interactions. However, it breaks down in the strongly attractive regime $\left(F_{2} \sim-1\right)$, where non-local interactions $F_{2}(q)$ are essential 2 . With these considerations, we arrive to the effective action

$$
S_{\text {eff }}=\int d s\left\{\left(s-\epsilon_{1}\right)\left(s-\epsilon_{2}\right)+w^{2}\right\}\left|\delta Q^{+}(s)\right|^{2}
$$

where $\epsilon_{1}=(1 / 25)\left(27+10 F_{2}\right), \epsilon_{2}=(1 / 25)\left(25+10 F_{2}\right)$ and $w=(1 / 25) \sqrt{20\left|F_{2}\right|}$ are real positive numbers in the vicinity of the EP. The zeros of the Lagrangian are given of course by Eq. S31.

In order to rewrite the effective action in the Hamiltonian formalism (first order in time), we introduce a two-component vector field $\delta Q^{+}=\left(\delta Q_{1}, \delta Q_{2}\right)$. In terms of this field, the effective action reads (please, see supplemental material for details)

$$
S_{\mathrm{eff}}=\int d s\left(\delta Q^{+}\right)^{\dagger}\left(s I-H_{\mathrm{eff}}\right) \delta Q^{+}
$$

where $I$ is the $2 \times 2$ identity matrix and the effective Hamiltonian is

$$
H_{\mathrm{eff}}=\left(\begin{array}{cc}
\epsilon_{1} & i w \\
i w & \epsilon_{2}
\end{array}\right)
$$

It is straightforward to verify that, integrating out the vector component $\delta Q_{2}$, we obtain the effective action of Eq. (S33) for the field $\delta Q_{1}$. Therefore, the dynamics near the singularity is driven by a $2 \times 2$ symmetric effective Hamiltonian (nonHermitian), which determines the properties of the $\mathrm{EP}^{40}$.

Hilbert space and topology- The Hilbert space spanned by the basis $\psi_{ \pm}$and its dual, spanned by $\phi_{ \pm}$, are in general different in non-Hermitian Hamiltonian systems. They are defined by

$$
\begin{aligned}
& H_{\text {eff }} \psi_{ \pm}=s_{ \pm} \psi_{ \pm} \\
& H_{\text {eff }}^{\dagger} \phi_{ \pm}=s_{ \pm}^{*} \phi_{ \pm} .
\end{aligned}
$$

Bi-orthogonality requires $\left\langle\phi_{i} \mid \psi_{j}\right\rangle=\delta_{i j}$ with $i, j= \pm$. Since the effective Hamiltonian is symmetric, the dual space is spanned by $\phi_{ \pm}=\psi_{ \pm}^{*}$. Solving Eq. (10), we find

$$
\psi_{ \pm}=c^{ \pm}\left(\begin{array}{c}
1 \\
\frac{-i}{\sqrt{1-z}}\left[1 \mp z^{1 / 2}\right]
\end{array}\right)
$$

where $c^{ \pm}$are complex normalization constants. We have introduced the variable $z=1+20 F_{2}$, in order to have the EP at $z=0$. As anticipated, not only $s_{+}=s_{-}$at the EP, but the eigenvectors collapse to $\psi_{ \pm}^{\mathrm{EP}}=c^{ \pm}(1,-i)$. This fact produces that $\left\langle\phi^{\mathrm{EP}} \mid \psi^{\mathrm{EP}}\right\rangle=0$, which is evidently in conflict with bi-orthogonality. In this way, the EP is a singularity in the structure of the Hilbert space ${ }^{8}$. This singularity induces remarkable topological properties. To show this, let us compute the geometric phase that the wave function picks up when the EP is winded in parameter space. For this, we analytically continue $z$ to the complex plane and define the Berry phase as $\gamma=i \oint_{C} d \ell \cdot \mathbf{A}$, where the one-form $\mathbf{A}=\left\langle\phi_{+} \mid \nabla \psi_{+}\right\rangle /\left\langle\phi_{+} \mid \psi_{+}\right\rangle \stackrel{41}{ }, C$ is a closed path and $\nabla$ is the gradient in parameter space $z$. The equivalent definition with $\phi_{-}$and $\psi_{-}$eigenvectors provides the same result. Notice that A is ill-defined at the EP since, at this point, the denominator is zero. The particular structure of the Hilbert space and its dual allows us to rewrite the vector form as a total derivative (locally a pure gauge), $\mathbf{A}=(1 / 2) \nabla \ln \left\langle\phi_{+} \mid \psi_{+}\right\rangle$. Thus, the EP is a branch point of the logarithm. Each time the phase of $\left\langle\phi_{+} \mid \psi_{+}\right\rangle$winds the branch point, the logarithm picks up a $2 \pi i$ term. This property does not depend on the specific path, provided the path encircles the EP. Thus, we can compute $\gamma$ considering a very small circumference around the EP. Using Eq. (12), we find for $|z|<<1,\left\langle\phi_{+} \mid \psi_{+}\right\rangle \sim z^{1 / 2}$. Due to the square-root singularity, the phase of $\left\langle\phi_{+} \mid \psi_{+}\right\rangle$is half the phase of $z$. Therefore, taken the path $C$ winding two times the EP, the Berry phase $\gamma=\pi$, in agreement with results obtained for general symmetric non-Hermitian Hamiltonians ${ }^{42}$. In this way, in encircling the EP, it is necessary to wind four times the singularity to return to the original state $\underline{\underline{43}}$. Recently, this unique topology of EPs was experimentally confirmed in metamaterials set ups 26,27 .

Experimental signatures- Information about collective excitations of strongly correlated systems can be obtained by measuring momentum-resolved dynamic susceptibility in the meV scale ${ }^{44}$. The detection of a stable mode near the usual zero sound could be an indication of the presence of an EP. Moreover, pump-probe spectroscopy $45-47$ yield important information on the dynamic response in the time domain. An experimental signature can be obtained from $\chi_{2}^{+}(\mathbf{q}, t)$, by Fourier transforming the DQS in the neighborhood of the EP. For $F_{2}>F_{2}^{c}$, the retarded susceptibility is

$$
\Re\left[\chi_{2}^{+}(\mathbf{q}, t)\right]=2 v_{F} q\left[\frac{\sin \left(\omega_{-} t\right)}{\omega_{-}}\right] \cos \left(\omega_{+} t\right) \Theta(t)
$$

where $\omega_{ \pm}=\left(s_{+} \pm s_{-}\right) v_{F} q / 2$ and $\Theta(t)$ is the Heaviside distribution. We clearly observe two well separated time scales since $\omega_{+} / \omega_{-}>>1$. At the EP, $\omega_{-}=0$ and $\sin \left(\omega_{-} t\right) / \omega_{-} \rightarrow$ $t$. Thus, the signature of the EP is a growing linear modulating function of time, $\chi_{2}^{+}(\mathbf{q}, t) \sim t \cos \left(\omega_{+} t\right)$. An approximate linear modulation can be observed on a huge range of intermediate times, even when the coupling is not fine tuned at $F_{2}=F_{2}^{c}$. On the other hand, for $F_{2}<F_{2}^{c}$, the dynamic response dramatically changes since the modulation is exponentially damped $\chi_{2}^{+}(\mathbf{q}, t) \sim \exp \left\{-\left|\omega_{-}\right| t\right\} \cos \left(\omega_{+} t\right)$. We depict these different regimes in Fig. 3 The abrupt change in the dynamical response at the EP should also be captured in quantum quench set ups $\underline{48}$. Another interesting possibility is to look for signatures on the AC electrical conductivity $\underline{49}$. 


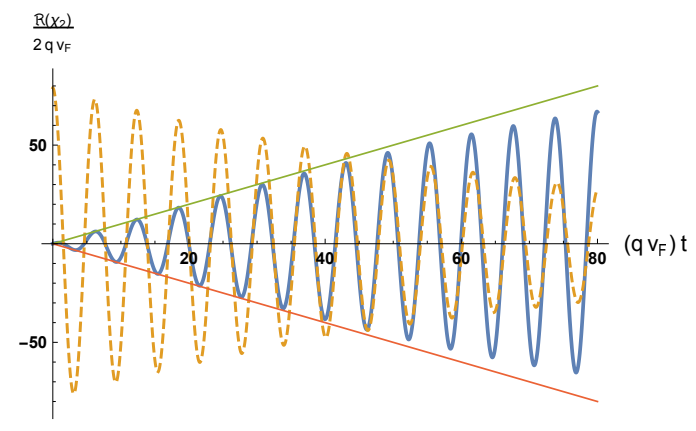

FIG. 3. $\Re\left[\chi_{2}(\mathbf{q}, t)\right] / 2 v_{F} q$ as a function of $\left(v_{F} q\right) t$. The continuous line is plotted with Eq. (13) by fixing $F_{2}=F_{2}^{c}+0.005$. The dashed line is the damped mode for $F_{2}=F_{2}^{c}-0.005$. The linear functions are the exact modulating function at the $\mathrm{EP}, F_{2}=F_{2}^{c}=-1 / 20$.

Summary and discussion - We have shown the existence of an EP in the collective mode spectrum of a Fermi liquid with weak attractive quadrupolar interactions. We completely characterize this singularity in terms of the Hilbert space structure as well as through its topological properties. We have also provided experimental signatures in the dynamical response. More complex models of Fermi liquids could lead to higher dimensional singularities, such as exceptional lines or surfaces 50,51 . For instance, if we consider isotropic density interactions $\left(F_{0}\right)$ in addition to the quadrupolar ones ${ }^{33}$, we still find square-root sigularities which, in the limit of small $F_{0}$, take the form $s_{+}-s_{-}=\sqrt{1+20 F_{2}+4 F_{0}}$. In this way, the spectrum has an exceptional line parametrized by $F_{2}+F_{0} / 5=-1 / 20$.

Concluding, non-Hermitian singularities appear in the spectrum of collective modes of Fermi liquids with higher angular momentum attractive interactions. Specific properties, such us the singularity location and dimensionality, are model dependent. However, its existence, its topological properties and experimental signatures are robust results. It could be important to investigate the influence of these singularities in the single quasi-particle spectrum and its effect on charge transport and other out-of-equilibrium properties.

\section{ACKNOWLEDGMENTS}

We would like to acknowledge Zochil González Arenas for useful comments. The Brazilian agencies, Fundação de Amparo à Pesquisa do Rio de Janeiro (FAPERJ), Conselho Nacional de Desenvolvimento Científico e Tecnológico (CNPq) and Coordenação de Aperfeiçoamento de Pessoal de Nivel Superior (CAPES) - Finance Code 001, are acknowledged for partial financial support. RA was partially supported by a $\mathrm{PhD}$ Fellowship from FAPERJ.
${ }^{1}$ F. P. Heinz-Peter Breuer, The theory of open quantum systems (Oxford University Press, New York, 2002).

2 I. Rotter and J. P. Bird, Reports on Progress in Physics 78, 114001 (2015)

3 T. Yoshida, R. Peters, and N. Kawakami, Phys. Rev. B 98, 035141 (2018)

4 Y. Michishita and R. Peters, Phys. Rev. Lett. 124, 196401 (2020)

${ }^{5} \mathrm{M}$. V. Berry and D. H. J. O'Dell, Journal of Physics A: Mathematical and General 31, 2093 (1998).

6 W. D. Heiss and A. L. Sannino, Journal of Physics A: Mathematical and General 23, 1167 (1990).

7 T. Kato, Perturbation theory for linear operators, Classics in Mathematics (Springer-Verlag, Berlin Heidelberg, 1995).

${ }^{8}$ U. Günther, I. Rotter, and B. F. Samsonov, Journal of Physics A: Mathematical and Theoretical 40, 8815 (2007).

9 T. Yoshida, R. Peters, N. Kawakami, and Y. Hatsugai, Phys. Rev. B 99, 121101 (2019)

${ }^{10}$ K. Kawabata, T. Bessho, and M. Sato, Phys. Rev. Lett. 123, 066405 (2019)

${ }^{11}$ K. Kawabata, K. Shiozaki, M. Ueda, and M. Sato, Phys. Rev. X 9, 041015 (2019)

${ }^{12}$ C. C. Wojcik, X.-Q. Sun, T. c. v. Bzdušek, and S. Fan, Phys. Rev. B 101, 205417 (2020)

${ }^{13}$ V. Kozii and L. Fu, "Non-hermitian topological theory of finitelifetime quasiparticles: Prediction of bulk fermi arc due to exceptional point," (2017), arXiv:1708.05841 [cond-mat.mes-hall]

${ }^{14}$ W. D. Heiss, M. Müller, and I. Rotter, Phys. Rev. E 58, 2894 (1998)

15 C. Jung, M. Müller, and I. Rotter, Phys. Rev. E 60, 114 (1999)

16 G. A. Álvarez, E. P. Danieli, P. R. Levstein, and H. M. Pastawski, The Journal of Chemical Physics 124, 194507 (2006) https://doi.org/10.1063/1.2193518

${ }^{17}$ H. Eleuch and I. Rotter, Phys. Rev. A 93, 042116 (2016)

N. Kaiser and W. Weise, The Nuclear Many-Body Problem, 1st ed., NATO Science Series 53 (Springer Netherlands, 2002).

19 H. Cartarius, J. Main, and G. Wunner, Phys. Rev. Lett. 99, 173003 (2007).

${ }^{20}$ M. Am-Shallem, R. Kosloff, and N. Moiseyev, Phys. Rev. A 93, 032116 (2016)

${ }^{21}$ L. Pan, S. Chen, and X. Cui, Phys. Rev. A 99, 011601 (2019)

${ }^{22} \mathrm{M}$. Aharonyan and E. G. D. Torre, Molecular Physics 117, 1971 (2019)

https://doi.org/10.1080/00268976.2019.1567849

${ }^{23}$ T. Yoshida, R. Peters, N. Kawakami, and Y. Hatsugai, Progress of Theoretical and Experimental Physics (2020), 10.1093/ptep/ptaa05s ptaa059, https://academic.oup.com/ptep/advance-article-pdf/doi/10.1093/ptep/pt

${ }^{24}$ C. Dembowski, H.-D. Gräf, H. L. Harney, A. Heine, W. D. Heiss, H. Rehfeld, and A. Richter, Phys. Rev. Lett. 86, 787 (2001).

25 A. D. Dente, R. A. Bustos-Marún, and H. M. Pastawski, Phys. Rev. A 78, 062116 (2008)

${ }^{26}$ M. Kang, W. Zhu, and I. D. Rukhlenko, Phys. Rev. A 96, 063823 (2017)

27 S. H. Park, S.-G. Lee, S. Baek, T. Ha, S. Lee, B. Min, S. Zhang, M. Lawrence, and T.-T. Kim, Nanophotonics , 20190489 (2020)

28 P. Nozieres and D. Pines, The Theory of Quantum Liquids (Perseus Books, 1999) p. 48.

1 V. Oganesyan, S. A. Kivelson, and E. Fradkin, Phys. Rev. B 64, 195109 (2001)

${ }^{30}$ E. Fradkin, S. A. Kivelson, M. J. Lawler, J. P. Eisenstein, and A. P. Mackenzie, Annual Review of Condensed Matter Physics 1, 153 (2010). 
http://www.annualreviews.org/doi/pdf/10.1146/annurev-conmatphys-070989-Vid3925Kogar, M. Mitrano, A. A. Husain, V. Mishra, M. S. Rak,

2 M. J. Lawler, D. G. Barci, V. Fernández, E. Fradkin, and L. Oxman, Phys. Rev. B 73, 085101 (2006)

32 D. G. Barci and D. Reyes, Phys. Rev. B 87, 075147 (2013)

${ }_{33}$ R. Aquino and D. G. Barci, Phys. Rev. B 100, 115117 (2019)

34 A. Klein, D. L. Maslov, L. P. Pitaevskii, and A. V. Chubukov, Phys. Rev. Research 1, 033134 (2019)

${ }^{35}$ M. P. Gochan, J. T. Heath, and K. S. Bedell, Journal of Physics: Condensed Matter 32, 345602 (2020)

${ }^{36}$ V. A. Zyuzin, P. Sharma, and D. L. Maslov, Phys. Rev. B 98, 115139 (2018)

37 A. Klein, S. Lederer, D. Chowdhury, E. Berg, and A. Chubukov, Phys. Rev. B 97, 155115 (2018)

38 J Nilsson and A. Phys. Rev. B 72, 195104 (2005)

39 W. H. Heiss and W. Steeb, Journal of Mathematical Physics 32, 3003 (1991). https://doi.org/10.1063/1.529044 L. Venema, P. D. Johnson, G. D. Gu, E. Fradkin, M. R. Norman, and P. Abbamonte, SciPost Phys. 3, 026 (2017)

45 C. Giannetti, M. Capone, D. Fausti, M. Fabrizio, F. Parmigiani, and D. Mihailovic, Advances in Physics 65, 58 (2016) https://doi.org/10.1080/00018732.2016.1194044

${ }^{46}$ F. Krausz and M. Ivanov, Rev. Mod. Phys. 81, 163 (2009)

${ }^{47}$ M. Mitrano, S. Lee, A. A. Husain, L. Delacretaz, M. Zhu, G. de la Peña Munoz, S. X.-L. Sun, Y. I. Joe, A. H. Reid, S. F. Wandel, G. Coslovich, W. Schlotter, T. van Driel, J. Schneeloch, G. D. Gu, S. Hartnoll, N. Goldenfeld, and P. Abbamonte, Science Advances 5 (2019), 10.1126/sciadv.aax3346. https://advances.sciencemag.org/content/5/8/eaax3346.full.pdf 48 N. Nessi, A. Iucci, and M. A. Cazalilla, Phys. Rev. Lett. 113, 210402 (2014)

49 J. Y. Khoo, P.-Y. Chang, F. Pientka, and I. Sodemann, Phys. Rev. B 102, 085437 (2020)

50 B. Zhen, C. W. Hsu, Y. Igarashi, L. Lu, I. Kaminer, ${ }^{40}$ H. Eleuch and I. Rotter, The European Physical Journal D 69, 229 (2015)A. Pick, S.-L. Chua, J. D. Joannopoulos, and M. Soljačić, 41 J. Garrison and E. Wright, Physics Letters A 128, 177 (1988). Nature 525, 354 (2015)

42 A. A. Mailybaev, O. N. Kirillov, and A. P. Seyranian, ${ }^{51}$ X. Zhang, K. Ding, X. Zhou, J. Xu, and D. Jin, Phys. Rev. A 72, 014104 (2005) Phys. Rev. Lett. 123, 237202 (2019)

${ }^{4}$ W. D. Heiss, The European Physical Journal D - Atomic, Molecular, Optical and Plasma Physics 7, 1 (1999) 


\section{Supplemental Material to "Exceptional points in Fermi liquids with quadrupolar interactions"}

\section{DYNAMIC QUADRUPOLAR SUSCEPTIBILITY}

In this section we give details of the calculation of the dynamic quadrupolar susceptibility (DQS) in a Fermionic model with local quadrupolar interactions. We begin by considering the simplest model of bi-dimensional spinless Fermions. The Hamiltonian is

$$
H=\int d^{2} r\left\{\psi^{\dagger}(\mathbf{r}) \epsilon(\nabla) \psi(\mathbf{r})+\frac{F_{2}}{4} \operatorname{Tr}\left[Q^{2}(\mathbf{r})\right]\right\}
$$

where $\psi(r)$ is a spinless Fermionic field operator. The bare dispersion relation is given by $\epsilon(\nabla)$, where $\nabla$ is the two-dimensional gradient operator. $F_{2}$ is the quadrupolar coupling constant. The quadrupolar Fermionic density

$$
Q_{i j}(\mathbf{r})=\psi^{\dagger}(\mathbf{r})\left[\nabla_{i} \nabla_{j}-\frac{\delta_{i j}}{2} \nabla^{2}\right] \psi(\mathbf{r})
$$

is a symmetric traceless tensor of $\operatorname{rank} 2 ; i, j=1,2$.

The dynamic quadrupolar susceptibility (DQS) is given by

$$
\chi_{i j l m}(\omega, \mathbf{q})=\left\langle Q_{i j}(-\omega,-\mathbf{q}) Q_{l m}(\omega, \mathbf{q})\right\rangle .
$$

However, the quadrupolar tensor has only two independent degrees of freedom, that can be parametrized by $m_{2}^{+}$and $m_{2}^{-}$in the following way

$$
Q=\left(\begin{array}{cc}
m_{2}^{+} & m_{2}^{-} \\
m_{2}^{-} & -m_{2}^{+}
\end{array}\right)
$$

Thus, among the sixteen components of Eq. $\underline{\mathrm{S} 3}$, only three are independent, say

$$
\begin{aligned}
\chi_{2}^{+}(\omega, \mathbf{q}) & =\left\langle m_{2}^{+}(-\omega,-\mathbf{q}) m_{2}^{+}(\omega, \mathbf{q})\right\rangle \\
\chi_{2}^{-}(\omega, \mathbf{q}) & =\left\langle m_{2}^{-}(-\omega,-\mathbf{q}) m_{2}^{-}(\omega, \mathbf{q})\right\rangle \\
\chi_{2}^{+-}(\omega, \mathbf{q}) & =\left\langle m_{2}^{+}(-\omega,-\mathbf{q}) m_{2}^{-}(\omega, \mathbf{q})\right\rangle
\end{aligned}
$$

Since the interaction is quartic in the Fermionic fields, the expectations values cannot be computed exactly. A computation using the traditional RPA approximation can be found in Ref. [S1]. In this paper, we describe the calculation using multidimensional bosonization, originally presented in Ref. [S2]. The latter approximation is accurate in a regime of small momentum $|\mathbf{q}|<<k_{F}$, where $k_{F}$ is the Fermi momentum. The result coincides with the former RPA approximation.

The essence of the bosonization approximation is to describe the effective dynamic of the system in a regime of energy and momentum very near the Fermi surface. In this sense, it is a low temperature $\left(k_{B} T<<\epsilon_{F}\right.$, where $\epsilon_{F}$ is the Fermi energy) and long distance $\left(q<<k_{F}\right)$ approximation. For weak interactions, this is the same regime of the Landau theory of Fermi liquids, then, it should reproduce the same results. The advantage of bosonization is that it is not limited to weak interactions.

It is by now well established that the dynamics induced the the Hamilotian of Eq. (S1) very near the Fermi surface can be entirely written in terms of a set of bosonic operators

$$
\delta n_{S}(\mathbf{r})=: \psi_{S}^{\dagger}(\mathbf{r}) \psi_{S}(\mathbf{r}):
$$

with $S=1, \ldots, N$. In this equation, $S$ labels the patch in which the Fermi surface was divided, and $\psi_{S}$ is the Fermionic operator with support on each small patch in momentum space; $i$. e. , $\psi_{S}(q)$ with $q<\lambda<\Lambda<<k_{S}$. The patch $S$ is a small rectangle centered at $\mathbf{k}_{F}=\mathbf{k}_{S}$ of width $\Lambda$ and height $\lambda$. The colons in Eq. (S8) means normal order with respect to a reference state with all the states inside the Fermi surface filled. At the end of the calculations the Fermi surface is reconstructed by taking the limit $\Lambda \rightarrow 0, N \rightarrow \infty$, with $N \Lambda=2 \pi k_{F}$. Within each patch, the Fermionic dispersion relation is linearized, $\epsilon(\mathbf{k})=\epsilon\left(\mathbf{k}_{S}\right)+\mathbf{v}_{S} \cdot \mathbf{q}$, where $v_{\mathbf{S}}$ is the Fermi velocity in each patch. This approximation is a good one in the Fermi liquid phase. However, near the Pomeranchuk regime, it should be corrected by curvature terms.

It is well known ${ }^{\mathrm{S} 2}$ that, the effective action of the system can be written in terms of the set of density fluctuations $\delta n_{S}(\omega, \mathbf{q})$. It can be split into a free and an interaction part, $S=S_{0}+S_{I}$. The free term is given by

$$
S_{0}=\int \frac{d^{2} q d \omega}{(2 \pi)^{3}} \sum_{S} \chi_{S}^{-1}(\omega, \mathbf{q}) \delta n_{S}(\omega, \mathbf{q}) \delta n_{S}(-\omega,-\mathbf{q})
$$


where the free density suceptibility is given by

$$
\chi_{S}(\omega, \mathbf{q})=\frac{\mathbf{v}_{S} \cdot \mathbf{q}}{\omega-\mathbf{v}_{S} \cdot \mathbf{q}}=\frac{\cos \left(\theta_{S}-\phi\right)}{s-\cos \left(\theta_{S}-\phi\right)}
$$

with $\mathbf{v}_{S}=v_{F}\left(\cos \theta_{S}, \sin \theta_{S}\right)$ and $\mathbf{q}=q(\cos \phi, \sin \phi)$. Thus, $\theta_{S}-\phi$ is the angle subtended between the momentum $\mathbf{q}$, and the Fermi velocity $\mathbf{v}_{S}$. In the last term of Eq. $\left(\mathbf{S 1 0}\right.$ we have introduced the dimensionless variable $s=\omega / v_{F} q$.

The interacting part of the action is given by,

$$
S_{I}=F_{2} \int \frac{d^{2} q d \omega}{(2 \pi)^{3}} \sum_{S, T} \cos \left[2\left(\theta_{S}-\theta_{T}\right)\right] \delta n_{T}(\omega, \mathbf{q}) \delta n_{S}(-\omega,-\mathbf{q})
$$

Note that the free, as well as, the interacting part of the action are quadratic in $\delta n_{S}$, then, it can be solved exactly. However, the calculation is not trivial since, while the free term is diagonal in the patch variable, the interaction term mixes patches.

The main goal of the present calculation is to write the action only in terms of the quadupolar moment, in order to read the corresponding susceptibility. To do this, we note that $\delta n_{S}$ is a periodic function of $\theta_{S}$, provided the Fermi surface is closed. Then, we can write the following Fourier expansion,

$$
\delta n_{S}(\mathbf{q})=m_{0}(\mathbf{q})+\sum_{\ell=1}^{\infty}\left\{m_{\ell}^{+}(\mathbf{q}) \cos \left(\ell \theta_{S}\right)+m_{\ell}^{-}(\mathbf{q}) \sin \left(\ell \theta_{S}\right)\right\}
$$

In is not difficult to check the transformation laws of each component $m_{\ell}^{ \pm}$. Since $\delta n_{S}$ is a scalar under rotations, it is immediate to see that $m_{0}$ is also a scalar and is related with the total density of the system. On the other hand, the couple $\left(m_{1}^{+}, m_{1}^{-}\right)$ transform as a vector under rotations and it is related with the total current. Moreover, $\left(m_{2}^{+}, m_{2}^{-}\right)$, does not transform as a vector but as the components of a symmetric traceless tensor as indicated in Eq. (S4], thus $m_{2}^{ \pm}$are the components of the quadrupolar moment.

The interaction term, $S_{I}$, only contains the quadrupolar components $m_{2}^{ \pm}$and it is simply given by

$$
S_{I}=\frac{F_{2}}{2} \int \frac{d^{2} q d \omega}{(2 \pi)^{3}}\left\{\left|m_{2}^{+}(\omega, \mathbf{q})\right|^{2}+\left|m_{2}^{-}(\omega, \mathbf{q})\right|^{2}\right\}
$$

that, it is evidently rotation invariant, since it is proportional to $\operatorname{Tr}\left(Q^{2}\right)$.

On the other hand, the free part of the action, $S_{0}$, is not diagonal in Fourier space and consequently mixes different momenta. Fortunately, since the interaction only contains $m_{2}^{ \pm}, S_{0}$ can be diagonalized and the components $m_{\ell}^{ \pm}$with $\ell \neq 2$, can be exactly integrated out, providing an action written only in terms of the quadrupolar components $m_{2}^{ \pm}$. An equivalent procedure is to consider just quadrupolar deformations

$$
\delta n_{S}(\mathbf{q})=m_{2}^{+}(\mathbf{q}) \cos \left(n \theta_{S}\right)+m_{2}^{-}(\mathbf{q}) \sin \left(n \theta_{S}\right)
$$

Replacing Eq. (S14) into Eq. $\left[\mathbf{S 9}\right.$ ) and integrating over the angles $\theta_{S}$, we obtain the effective action in terms of the quadrupolar momentum $m_{2}^{ \pm}$. In a covariant notation we find,

$$
S=\frac{1}{2} \int \frac{d^{2} q d \omega}{(2 \pi)^{3}}\left\{\left[\Gamma_{0}(s)-F_{2}\right] \operatorname{Tr} \hat{Q}^{2}-\Gamma_{4}(s) \operatorname{Tr}(\hat{P} \hat{Q})^{2}\right\}
$$

where the tensor $\hat{Q}$ is given by Eq. (S4) and we have introduced a second order tensor $\hat{P}$ given by

$$
\hat{P}=\left(\begin{array}{cc}
\cos (2 \phi) & \sin (2 \phi) \\
\sin (2 \phi) & -\cos (2 \phi)
\end{array}\right)
$$

The coefficients $\Gamma_{0}$ and $\Gamma_{4}$ are given in terms of the free susceptibilies,

$$
\begin{aligned}
& \Gamma_{0}(s)=\frac{\chi_{0}^{0}(s)}{\left(\chi_{0}^{0}(s)\right)^{2}-\left(\chi_{0}^{4}(s)\right)^{2}} \\
& \Gamma_{4}(s)=\frac{\chi_{4}^{0}(s)}{\left(\chi_{0}^{0}(s)\right)^{2}-\left(\chi_{0}^{4}(s)\right)^{2}}
\end{aligned}
$$


where,

$$
\chi_{0}^{\ell}(s)=\int \frac{d \theta}{2 \pi} \frac{\cos \theta}{s-\cos \theta} e^{i \ell \theta}
$$

Integrating this expression for $s>1$ and analytically continuing for $s<1$ we find in particular,

$$
\begin{aligned}
& \chi_{0}^{0}(s)=-1+K_{0}(s) \\
& \chi_{0}^{4}(s)=K_{0}(s)\left(\frac{1-K_{0}(s)}{1+K_{0}(s)}\right)^{2}
\end{aligned}
$$

with

$$
K_{0}(s)=\frac{s}{\sqrt{s^{2}-1}}
$$

We can also explicitly re-write the action of Eq. (S15) in a non-covariant form, in terms of $m_{2}^{ \pm}$finding,

$$
\begin{gathered}
S=\int \frac{d^{2} q d \omega}{(2 \pi)^{3}}\left\{\left(\Gamma_{0}(s)-\Gamma_{4}(s) \cos (4 \phi)-F_{2}\right)\left|m_{2}^{+}\right|^{2}+\left(\Gamma_{0}(s)+\Gamma_{4}(s) \cos (4 \phi)-F_{2}\right)\left|m_{2}^{-}\right|^{2}\right. \\
\left.-\Gamma_{4}(s) \sin (4 \phi)\left[m_{2}^{+} m_{2}^{-*}+m_{2}^{+*} m_{2}^{-}\right]\right\}
\end{gathered}
$$

This is the effective action for the quadrupolar moments $m_{2}^{ \pm}(\omega, \mathbf{q})$. Due to the tensor character of $m_{2}^{ \pm}$, the action explicitly depends on the angle $\phi$. For arbitrary direction, the action is not diagonal in the components $m_{2}^{ \pm}$. We can gain more intuition by fixing a particular direction, for instance $\mathbf{q}=q \hat{i}$, i. e. , $\phi=0$. In this case, the Lagrangian density has the simpler diagonal form,

$$
\mathcal{L}=\left(\Gamma_{0}(s)-\Gamma_{4}(s)-F_{2}\right)\left|m_{2}^{+}(\omega, \mathbf{q})\right|^{2}+\left(\Gamma_{0}(s)+\Gamma_{4}(s)-F_{2}\right)\left|m_{2}^{-}(\omega, \mathbf{q})\right|^{2}
$$

from which we can read the longitudinal and transverse inverse quadrupolar susceptibilities

$$
\left(\chi_{2}^{ \pm}\right)^{-1}=\Gamma_{0}(s) \mp \Gamma_{4}(s)-F_{2}
$$

Inverting this expression and using Eqs. (S17) and (S18) we finally find,

$$
\chi_{2}^{ \pm}(\omega, \mathbf{q})=\frac{\chi_{0}^{0}(s) \pm \chi_{4}^{0}(s)}{1-F_{2}\left(\chi_{0}^{0}(s) \pm \chi_{4}^{0}(s)\right)}
$$

This result coincides with that presented in Refs. $\mathrm{S1}$ and $\mathrm{S2}$ and with Eq. (2) o the main paper.

\section{EFFECTIVE ACTION NEAR THE EXCEPTIONAL POINT}

The effective Lagrangian of Eq. (S24) is highly non-local in time. In fact, it has a cut for $s<1$. In order to have a simpler expression, let us expand the longitudinal part around the branch point $s=1$. We introduce a small variable $z=\sqrt{(s-1) / 2}$. In term of $z$, we can simplify $K_{0}(s)$ given by Eq. (S22),

$$
K_{0}(s) \sim \frac{1}{2} \frac{1}{z}
$$

Within this approximation

$$
\chi_{0}^{0}+\chi_{0}^{4} \sim \frac{1}{z}-5-4 z+O\left(z^{2}\right)
$$

Replacing this expression in the longitudinal part of the Lagrangian we find the kernel

$$
\mathcal{L}^{+}=\Gamma_{0}(s)-\Gamma_{4}(s)-F_{2} \sim-F_{2}+z+5 z^{2}+O\left(z^{3}\right)
$$

Turning back to the original variables we obtain

$$
\mathcal{L}^{+}(s)=-F_{2}+\sqrt{\frac{s-1}{2}}+5\left(\frac{s-1}{2}\right)+O\left((s-1)^{3 / 2}\right) .
$$


which coincides with Eq. (4) of the paper.

We are interested in the zeros of $\mathcal{L}^{+}(s)$. Therefore, solving $\mathcal{L}^{+}(s)=0$ we obtain

$$
s_{ \pm}=\frac{1}{25}\left\{\left(26+10 F_{2}\right) \pm \sqrt{20 F_{2}+1}\right\} .
$$

Note that the zeros are generally order one, except at $F_{2}=F_{c}=-1 / 20$, where both zeros are degenerate $s_{+}=s_{-}=51 / 50>$ 1. For $F_{2}<F_{c}$, the zeroes are complex conjugates. Since for $F_{2}>F_{c}, s_{ \pm}>1$, the kernel $\mathcal{L}^{+}(s)$ should be an analytic function of $s$ in a neighborhood of these points. Thus, we can write

$$
\mathcal{L}^{+}(s)=\left(s-s_{+}\right)\left(s-s_{-}\right)
$$

However, it is convenient to re-write this expression in terms of real positive numbers. Using the explicit expression for $s_{ \pm}$, given by Eq. S31) we easily find,

$$
S_{\mathrm{eff}}=\int d s\left\{\left(s-\epsilon_{1}\right)\left(s-\epsilon_{2}\right)+w^{2}\right\}\left|m_{2}^{+}(s, \mathbf{q})\right|^{2}
$$

where we have defined

$$
\begin{aligned}
\epsilon_{1} & =\frac{1}{25}\left(27+10 F_{2}\right) \\
\epsilon_{2} & =\frac{1}{25}\left(25+10 F_{2}\right) \\
w & =\frac{1}{25} \sqrt{20\left|F_{2}\right|}
\end{aligned}
$$

Therefore, near the singular point $s_{+} \sim s_{-}$, the longitudinal component of the quadrupolar momentum satisfy the following second order equation

$$
\left\{\left(s-\epsilon_{1}\right)\left(s-\epsilon_{2}\right)+w^{2}\right\} m_{2}^{+}(s, \mathbf{q})=0
$$

Near the EP, $s \neq \epsilon_{2}$, then we can write the equation as

$$
\left\{\left(s-\epsilon_{1}\right)-i w\left(\frac{i w}{s-\epsilon_{2}}\right)\right\} m_{2}^{+}(s, \mathbf{q})=0
$$

Introducing the variables

$$
\begin{aligned}
& \delta Q_{1}=m_{2}^{+} \\
& \delta Q_{2}=\frac{i w}{s-\epsilon_{2}} m_{2}^{+}
\end{aligned}
$$

we immediately find

$$
\begin{aligned}
& \left(s-\epsilon_{1}\right) \delta Q_{1}-i w \delta Q_{2}=0 \\
& \left(s-\epsilon_{2}\right) \delta Q_{2}-i w \delta Q_{1}=0
\end{aligned}
$$

These first order system of equations can be obtained from a Hamiltonian formalism. By introducing a two-component vector field $\delta Q^{+}=\left(\delta Q_{1}, \delta Q_{2}\right)$ the effective action is written as

$$
S_{\mathrm{eff}}=\int d s\left(\delta Q^{+}\right)^{\dagger}\left(s I-H_{\mathrm{eff}}\right) \delta Q^{+}
$$

where $I$ is the $2 \times 2$ identity matrix and the effective Hamiltonian is

$$
H_{\mathrm{eff}}=\left(\begin{array}{cc}
\epsilon_{1} & i w \\
i w & \epsilon_{2}
\end{array}\right)
$$

as shown in Eq. (9) of the main paper.

[S1] V. Oganesyan, S. A. Kivelson, and E. Fradkin, Phys. Rev. B 64, 195109 (2001),

[S2] M. J. Lawler, D. G. Barci, V. Fernández, E. Fradkin, and L. Oxman, Phys. Rev. B 73, 085101 (2006) 